\title{
Main characteristics of the fast disruption mitigation valve
}

S. A. Bozhenkov, K.-H. Finken, M. Lehnen, and R. C. Wolf

Citation: Review of Scientific Instruments 78, 033503 (2007);

View online: https://doi.org/10.1063/1.2712798

View Table of Contents: http://aip.scitation.org/toc/rsi/78/3

Published by the American Institute of Physics

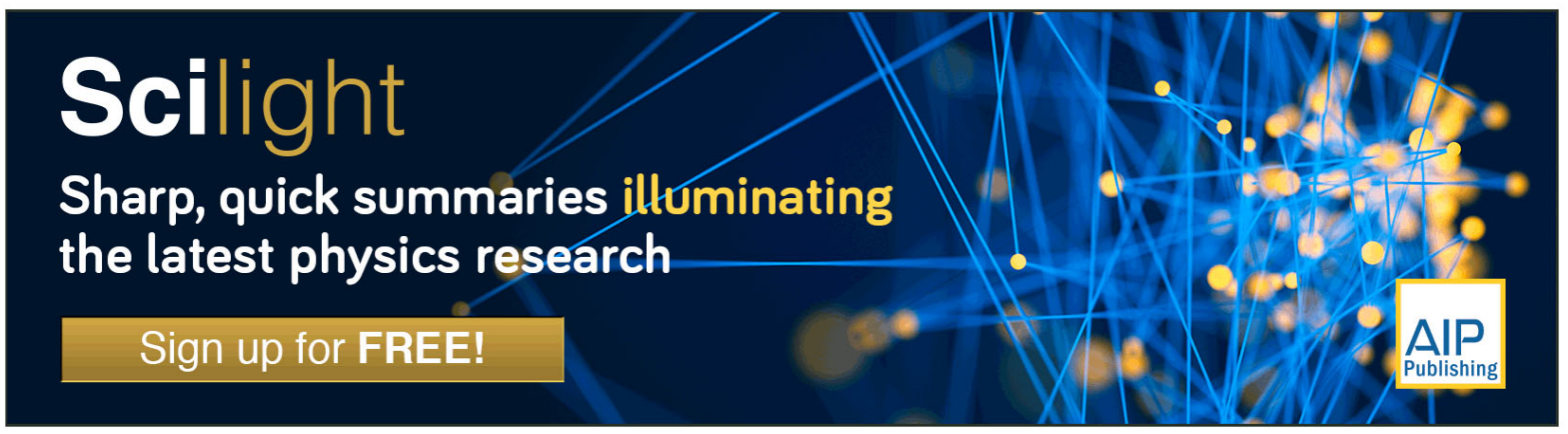




\title{
Main characteristics of the fast disruption mitigation valve
}

\author{
S. A. Bozhenkov, ${ }^{\text {a) }}$ K.-H. Finken, M. Lehnen, and R. C. Wolf \\ Institute of Plasma Physics, Forschungzentrum Juelich GmbH, EUROATOM-Association, Trilateral Euregio \\ Cluster, D-52425 Juelich, Germany
}

(Received 6 December 2006; accepted 30 January 2007; published online 15 March 2007)

\begin{abstract}
The article presents a detailed investigation of the fast disruption mitigation valve developed at FZJ Juelich. The essence of this study is the novel direct observation of the piston motion by means of a fast framing camera. The piston stroke and the injection duration are shown to strongly depend on the operational pressure and the used gas. The same is true for the valve throughput. The analysis revealing the leading contribution of the injection duration in this modification is given. The knowledge of the injection duration is also used to reconstruct the characteristic pressure decay rates and the gas outflow rates. The means to increase the gas outflow are discussed. The main found valve characteristics are: (1) valve reaction time, i.e., the delay between the application of the trigger signal and the achievement of reliably observable opening $0.5 \mathrm{~mm}$, is about $0.3 \mathrm{~ms}$; (2) the maximum achieved throughput is 7.5 bar 1 for argon and 9.5 bar 1 for helium; (3) the maximum

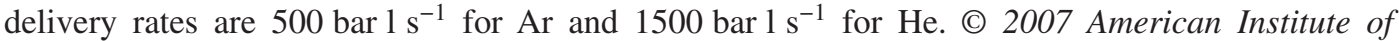
Physics. [DOI: 10.1063/1.2712798]
\end{abstract}

\section{INTRODUCTION}

The operation of tokamaks is prone to fast accidental losses of the stored energy (tens of MJ in present-day machines). These losses referred to as disruptions occur in two stages. In the first stage the whole plasma thermal energy is deposited onto the limited area in contact with the plasma on a time scale shorter than $1 \mathrm{~ms}$. After that the current flowing through the several electron-volt cold plasma decays in a resistive way, with the characteristic time constant $\tau=L / R$ being of the order of $10 \mathrm{~ms}$. It is worth pointing out that the stored magnetic energy associated with the plasma current in tokamaks is even higher than the thermal inventory. The disruptions are highly undesirable since they result in extreme loads on the machine usually categorized in the following groups: $^{1}$ (i) excessive heat loads; (ii) a local energy deposition by a beam of mega-electron-volt electrons (runaway electrons); (iii) electromagnetic forces resulting from the closing of the plasma current inside the vessel (halo currents). The loads being thought to become critical and to effectively limit the lifetime of the machine in the future fusion experiment ITER (the full stored energy about $1 \mathrm{GJ}$, the energy gain factor 10$),{ }^{2}$ an elaborated technique protecting a tokamak during disruption phase has to be developed.

The most promising technique mitigating all negative consequences of a disruption is a fast massive noble gas injection. A good radiating impurity decreases the heat flux density by redistributing the energy over a larger surface area. The created high particle density provides the collision frequency high enough to hinder runaway acceleration. At the same time the resulting high plasma resistivity makes the current decay faster, thus limiting the currents transferred to the vessel walls. The required amount of particles is given by

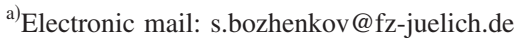

the energy content of the tokamak. In the present day experiments it equals to about 10 bar $1\left(1\right.$ bar $1 \approx 2.4 \times 10^{22}$ particles) to be injected in the characteristic time of $10 \mathrm{~ms}$. This number is to be increased for ITER, however, the exact scaling is still under discussion. As potential gases to be puffed the following are considered: $\mathrm{He}, \mathrm{Ne}$, and $\mathrm{Ar}$. A high $\mathrm{Z}$ impurity has an advantage of the higher radiative cooling rate while the lighter atoms are able to provide higher influx rates. Among the mentioned gases, helium possesses the highest flow rate, however, the total amount of the injected helium is limited by the presence of helium cooled cryopumps in tokamak structures. To sum up, a gas puff system for disruption mitigation must meet the following requirements: the system reaction time is about $1 \mathrm{~ms}$, the total amount of injected particles is more than 10 bar 1 and the injection rate is of the order of 1000 bar $1 \mathrm{~s}^{-1}$. The injector operational pressure as found from the last two criteria and the assumption of a reasonable size is in the range 10-50 bar.

The systems used for the gas injection are either solenoid-actuated valves or the valves activated by eddy currents. It is to be mentioned that there are also attempts to use piezoelectric valves. However, the gas flow rate $\sim 1$ bar $1 \mathrm{~s}^{-1}$ achieved with such systems ${ }^{3,4}$ is much lower than required for the large scale experiments. The high performance solenoid valves extensively used at DIII-D, ${ }^{5,6}$ Alcator-C, ${ }^{7}$ and Tore-Supra ${ }^{8}$ are similar to that described in Ref. 9, in which the ferromagnetic piston is drawn by the gradient of the magnetic field. In the recently emerged two stage valve ${ }^{10}$ the solenoid valve is the pilot one, the second stage being opened by the pressure imbalance created by the outflow from the first stage. Though providing in both mentioned cases the valve opening time shorter than a millisecond the ferromagnetic piston does not allow the valve to be installed in the immediate vicinity of a tokamak. A several meter long tube connecting the injection system and the vessel should be 

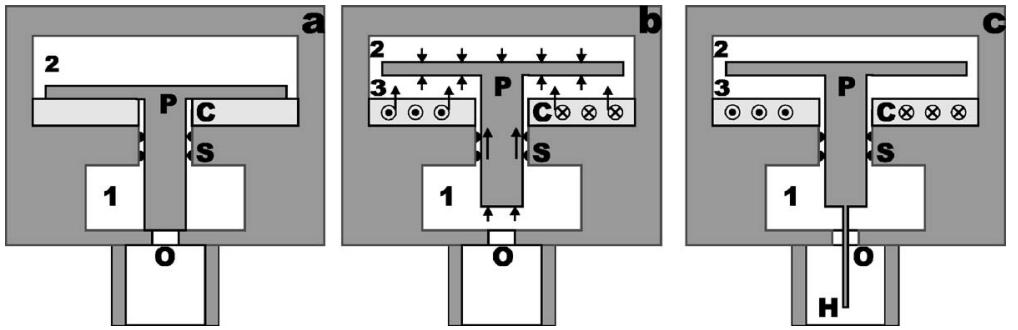

FIG. 1. The schematic view of the valve. (a) the valve is charged; (b) the valve is being opened, main forces are illustrated; and (c) the piston modified for the position registration. The notation: 1 -the chamber containing the gas to be injected (working chamber); 2 -the chamber filled with the gas providing the closing force (backchamber); 3-part of the backchamber separated by the piston; $P$-the aluminum piston; $C$-the pancake coil; $S$-the sealing separating working and backchambers; $O$ - the orifice; and $H$-the piston extension.

introduced. This fact considerably increases the total system reaction time consisting of the valve opening time and the time for the gas to travel through the delivery tubes. As opposed to it, the disruption mitigation valve (DMV) activated by eddy currents ${ }^{11}$ does not exploit any ferromagnetic materials and can be reliably operated in a high static magnetic field as it has been already demonstrated. ${ }^{12,13}$ This type of DMV is currently used at the tokamak TEXTOR and has been recently installed at the largest fusion experiment JET. The analysis of these experiments as well as the possible ITER application require a better understanding and detailed calibration data of the DMV.

The present article describes a novel method of the valve characterization. The method is based on the direct observation of the piston motion by means of the fast framing camera. The results of these observations are used to determine the valve reaction time, the gas outflow rates and to explain dependence of the valve performance on the operational parameters.

\section{DMV AND EXPERIMENT DESCRIPTION}

To begin with, briefly consider an ideal DMV working cycle. In the charged state [Fig. 1(a)] the aluminum mushroom-shaped piston, with the small and back diameters 20 and $200 \mathrm{~mm}$ correspondingly, effectively closes the 10 $\mathrm{mm}$ orifice of the working chamber owing to the pressure imbalance on the orifice surface. The registered leak rate does not exceed $10^{-5}$ bar $1 \mathrm{~s}^{-1}$ as required by a tokamak vacuum system. In the discussed valve the volume of the working chamber is $320 \mathrm{ml}$, which differs from that used at TEXTOR and JET: 30 and $650 \mathrm{ml}$. The scalings of the obtained results with volume are straightforward. The pressures in working $\left(p_{w}\right)$ and back $\left(p_{b}\right)$ chambers are in the range 5-60 bar and related by the closing condition $p_{w}<1.8 p_{b}$ given by the ratio of the actual areas $A_{1}, A_{2}$ on which the pressures are acting, as it is illustrated in Fig. 2. When the capacitance bank $(C \approx 200 \mathrm{mF}, U \approx 2 \mathrm{kV})$ is discharged

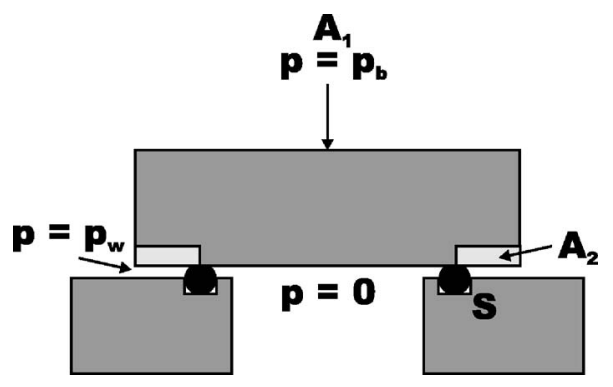

FIG. 2. The detailed view of the orifice. The notation: $S$-the O-ring sealing the orifice; $A_{1}, A_{2}$-the areas on which the $p_{b}$ and $p_{w}$ are acting. through the coil a time-varying magnetic field excites eddy currents in the back part of the piston. The resulting $j \times B$ force opens the valve by repelling the piston [Fig. 1(b)]. A typical timescale of the described process is about $0.5 \mathrm{~ms}$ as it is illustrated by the oscillogram of the current flowing through the coil (Fig. 3). Laboratory measurements indicate that about $5 \%$ of the stored electrostatic energy can be transformed into kinetic energy of the piston.

In reality the piston dynamics is affected by all applied forces illustrated in Fig. 1(b): EM force exerted by the coil current, pressures $p_{1} \equiv p_{w}, p_{2}, p_{3}$ and the friction force in the sealing separating the chambers (the pressure equivalent of the friction force $p_{\mathrm{fr}} \equiv F_{\mathrm{fr}} / A_{1}$ is 5 bar), where the pressures are themselves dependent on the piston movement. It is this dynamics that determines the width and the duration of the valve opening and, hence, the gas injection rate and the total amount of the puffed gas. To measure the opening characteristics of the valve experimentally a $3 \mathrm{~mm}$ thin pin was mounted at the leading end of the piston as it is sketched in Fig. 1(c). The displacement of the pin was observed with the aid of the fast framing camera PSI $5^{14}$ through a vacuum window. The optical setup provided a spatial resolution of about $0.3 \mathrm{~mm}$. This approach allows the opening characteristics to be measured with a pressure evolution very similar to the original one when the piston extension is not mounted. Furthermore, the average gas outflow rate is easy to estimate from the obtained injection durations without implementing any particular scheme of the unsteady transonic flow measurements. The disturbances introduced by the presence of the pin are thought to be insignificant: piston mass modification is about $1.7 \%$, the orifice area change is about $9 \%$.

Injections were performed into a $201 \mathrm{vol}$ evacuated to

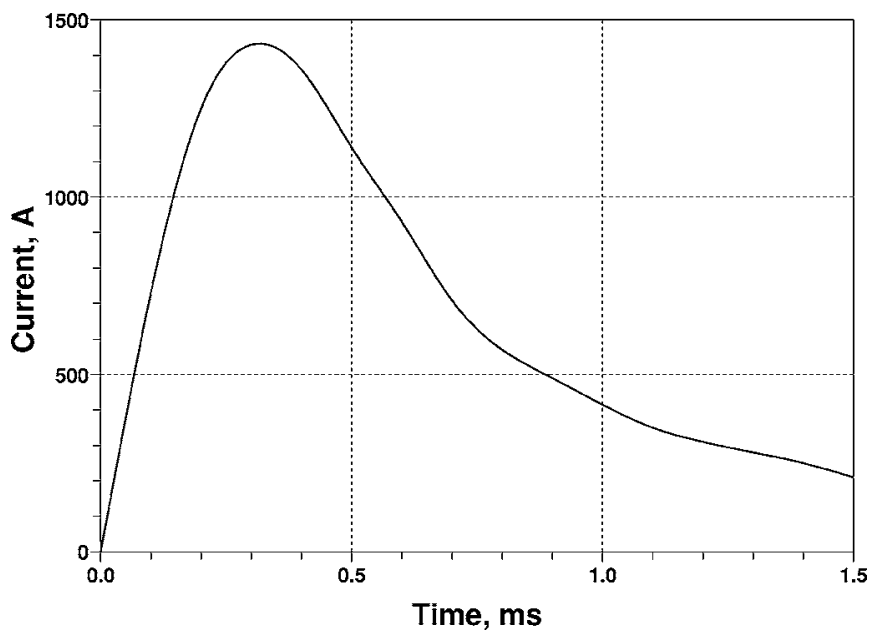

FIG. 3. The typical oscillogram of the current flowing through the coil. 


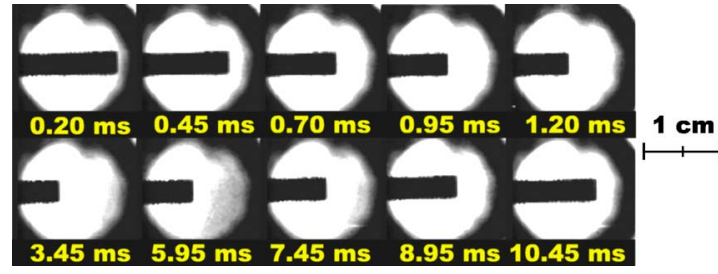

FIG. 4. An example of a recorded frame sequence showing the motion of the pin. The pin is a black rectangle observed in a backillumination. The valve located on the left is not shown. The corresponding frame times relative to the trigger signal are denoted.

$\sim 10^{-2}$ Torr. In addition to the piston position registration the pressures in the working and back sections were measured before and after every experiment by means of pressure transducers. During the tests, only helium and argon were addressed as being of particular interest for the disruption mitigation experiments. The gases in the back and working sections could be the same or different, i.e., the following configurations of gases were used: "Ar/Ar," "He/He," "Ar/ $\mathrm{He}$," and "He/Ar," where the first symbol stands for the working gas and the last one is the gas in the back section. The backpressure $p_{b}$ was varied in the range 5-35 bar, the working one was either equal to that $p_{w}=p_{b}$ ("equal") or higher by the fixed factor $p_{w}=1.5 p_{b}$ ("overpressure").

\section{OPENING CHARACTERISTICS}

An example of the recorded frame sequence of the pin movement is shown in Fig. 4. Upon receiving the trigger signal at time moment 0 the piston together with the pin are drawn to the left: the valve is opened. As the pressure in the working chamber decays the resulting decelerating force makes piston stop and move back. The original set consists of 300 frames obtained with $50 \mu$ s time resolution. The piston position relative to the closed state as a function of time is given in Fig. 5. The noticeable opening of $0.5 \mathrm{~mm}$ to be associated with the reaction time of the system is achieved about $0.3 \mathrm{~ms}$ after the trigger in all cases. The full opening

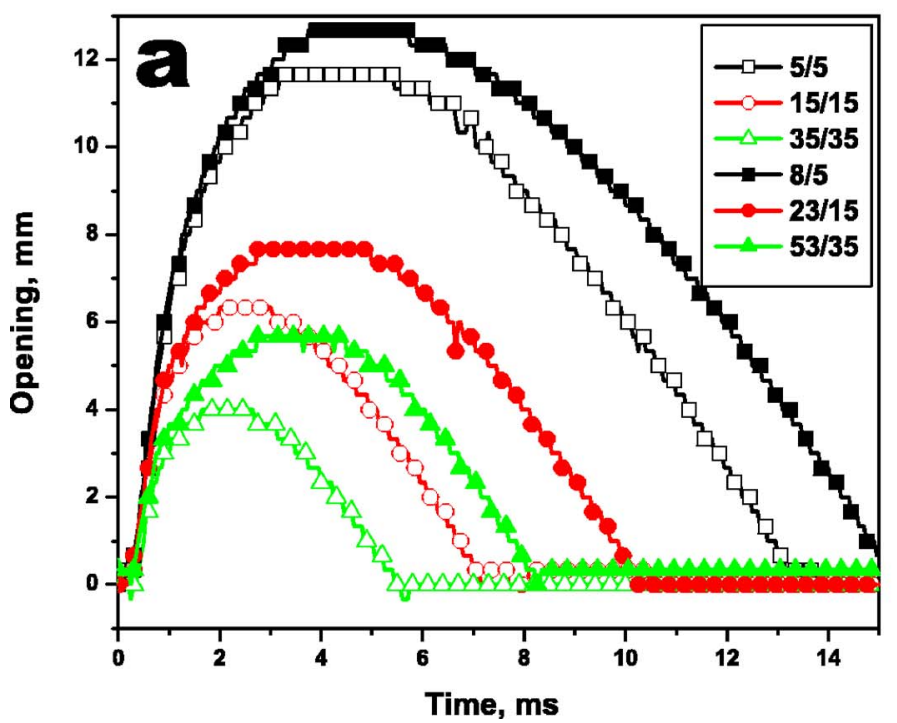

occurs after approximately 1-2 ms. The detailed dynamics depends on the gas species and the overpressure factor as it is discussed later.

The graph in Fig. 5(a) compiles the time-position correspondences for a number of the used pressures in $\mathrm{He} / \mathrm{He}$ experiments. The curves are labeled by the corresponding working and backpressures " $p_{w} / p_{b}$ ". It can be concluded from the plot (curves " $5 / 5$," "15/15," and " $35 / 35$ ") that an increase of the backpressure decreases the opening width and shortens the opening duration. While the overpressure (curves " $8 / 5$," "23/15," and "53/35") provides wider gap and prolongs the injection time by keeping the backpressure balanced for a longer period. The described results are in full correspondence with the change of the term $p_{b}-p_{w}(t)$ in the equation of the piston motion

$$
m_{p} \frac{d V}{d t}=\left[p_{b}-p_{w}(t)-p_{f r} \operatorname{sign}(V)\right] A_{1},
$$

where $A_{1}$ is the active surface area. The backward motion starts at the time moment when the backpressure exceeds the sum of the working and friction pressures. Since the gas outflow rate is expected to grow with $p_{w}$, this motion and the valve closing take place earlier at higher operational pressures.

The similar dependencies are observed in the case of Ar/Ar experiments [Fig. 5(b)]. However, in comparing plots of Figs. 5(a) and 5(b) the following two points are to be discussed. First, injection times in $\mathrm{Ar} / \mathrm{Ar}$ experiments are comparable to that in $\mathrm{He} / \mathrm{He}$ set in spite of the expected slower decrease of $p_{w}$. The gas outflow velocity being of the order of sound speed $c_{s}$ is about three times less in the case of argon injection. And second, the piston is stopped much earlier and consequently the achieved valve opening is narrower. The explanation is likely to be related to the gas dynamics in the back section. Indeed, the time required for the equilibration of the argon pressure along both sides of the piston backpart is found from the ratio of the back radius $r$ to the sound speed $c_{s}$. This value amounts to about $0.3 \mathrm{~ms}$ to be

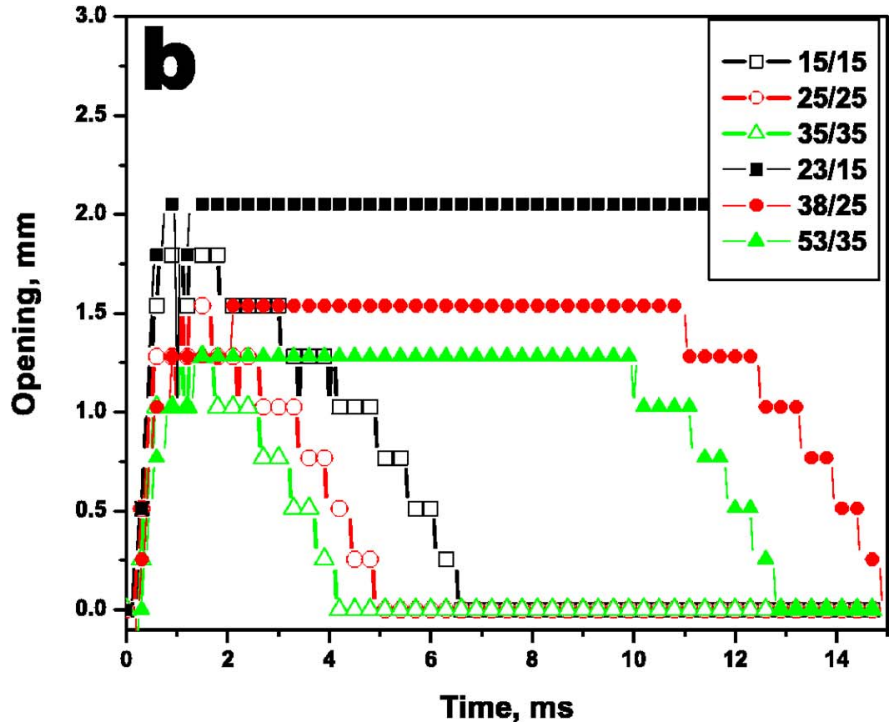

FIG. 5. The dependence of the piston position on time for different pressures. (a) He/He and (b) Ar/Ar. The pressures in the legends are indicated in the format $p_{w} / p_{b}$. The cases of $p_{w}=p_{b}$ (equal) and $p_{w}=1.5 p_{b}$ (overpressure) are shown. 

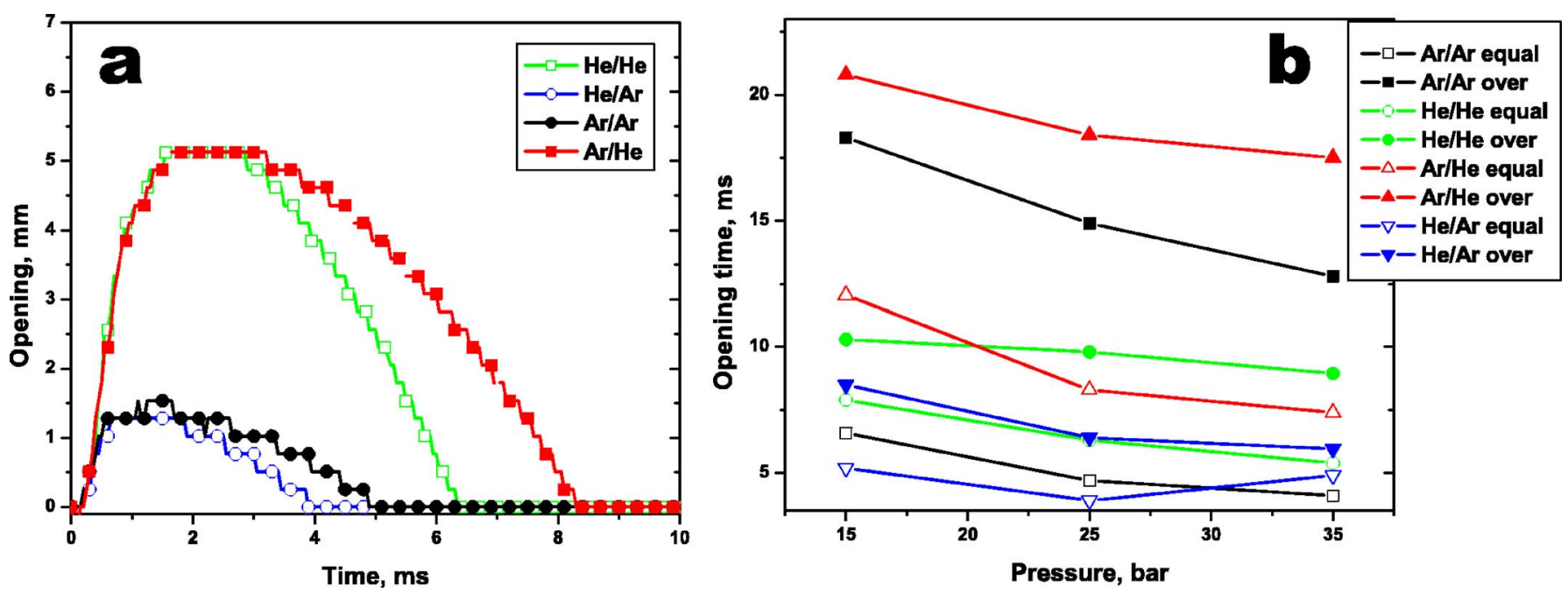

FIG. 6. The effect of using He in the back chamber. (a) the comparison of the piston position for different combinations of gases $p_{w}=p_{b}=25$ bar and (b) the comparison of the injection durations for different pressures and gasses. The notation: "the working gas/the gas in the backchamber"; "equal" $-p_{w}=p_{b}$; "over" $-p_{w}=1.5 p_{b}$.

compared with the characteristic time of the problem $0.5 \mathrm{~ms}$. Moreover the $1 \mathrm{~mm}$ narrow and $3 \mathrm{~mm}$ long separation gap between subsections 2 and 3 (Fig. 1) is to aggravate the imbalance. The overpressure better evens the forces and provides the time for the backpressure relaxation, which leads to a dramatic performance improvement to be shown later. In helium experiments the pressures in the backpart are effectively equilibrated.

The described analysis leads us to conclude that helium is to be preferred in the back section. Confirming this conclusion is the other series of measurements performed with the distinct gases in the chambers: $\mathrm{Ar} / \mathrm{He}$ and $\mathrm{He} / \mathrm{Ar}$. Figure 6(a) shows the comparison of the time-position dependencies for all gas combinations at the fixed working pressure $p_{w}$ $=p_{b}=25$ bar. Reformulating the earlier made conclusion once more, helium in the back section provides the wider valve opening and prolongs the injection time. The difference in the slopes of the curves $\mathrm{He} / \mathrm{He}$ and $\mathrm{Ar} / \mathrm{He}$ (as well as $\mathrm{He} / \mathrm{Ar}$ and $\mathrm{Ar} / \mathrm{Ar}$ ) is caused by the difference in sound velocities giving rise to the unequal decay rates. It is to be noted that the overpressure effect is in general the same for any gas combination and it is not illustrated here to clarify the plot.

To perform estimations of the gas flow rates the injection durations were determined [Fig. 6(b)]. The accuracy of the data is specified mainly by the spatial resolution and constitutes approximately $0.35 \mathrm{~ms}$. The end of the injection was not directly observed in the "Ar/He over" case due to the limited record length. The values were found by extrapolating the time-position curves with a second order polynomial. All main findings are clearly seen in this figure: the duration decreases as the pressure increases and the choice of the gas combination and overpressure factor plays a significant role. The argon tests appear to be more sensitive to the overpressure effect.

To sum up, to achieve better performance of the DMV it is advised to use helium in the back section and the overpressure in the working chamber.

\section{VALVE EFFICIENCY, THROUGHPUT, AND DECAY RATE}

Before proceeding to measurements, the appropriate terminology is to be introduced. The simplest model of the gas outflow with the sound velocity $c_{s}$ from the volume $V$ through the hole of area $S_{o}$ leads to an exponential law of the particle content evolution

$$
\frac{d N}{d t} \approx-N \frac{c_{s} S_{0}}{V} .
$$

That is $N=N(0) \exp \left(-\alpha_{\mathrm{id}} t\right)$, where $\alpha_{\mathrm{id}}=c_{s} \cdot S_{o} / V$ is the ideal decay rate. It is reasonable to characterize the process by measuring $\alpha$. The value reciprocal to the decay rate corresponds to the characteristic volume exhaust time. The a priori estimation for the used DMV gives decay rates of $0.22 \mathrm{~ms}^{-1}$ for helium and of $0.07 \mathrm{~ms}^{-1}$ for argon, or approximately 5 and $14 \mathrm{~ms}$ in terms of the exhaust times. The validity of the model has to be proven in the course of the analysis.

Let us first consider the valve efficiency. The dimensionless efficiency is defined as the amount of the injected particles relative to the initial particle content in the working chamber

$$
\mathrm{Ef}=\frac{p_{w}(0)-p_{w}(\infty)}{p_{w}(0)}=1-\exp \left(-\alpha t^{*}\right)
$$

where $t^{*}$ is the injection duration. The efficiency itself is easy to obtain, however, in this article the simultaneous measurements of the injection time reveal the reasons of the observed tendencies. The results of the efficiency measurements are presented in Fig. 7(a) for helium and Fig. 7(b) for argon. It can be concluded from the plots that the use of the overpressure or helium in the back section improves the efficiency by a factor between 1.5 and 3, the exact number depending on the particular conditions. There are only two ways to modify the efficiency either by changing the duration of the injection or by modifying the decay rate. The comparison of the injec- 

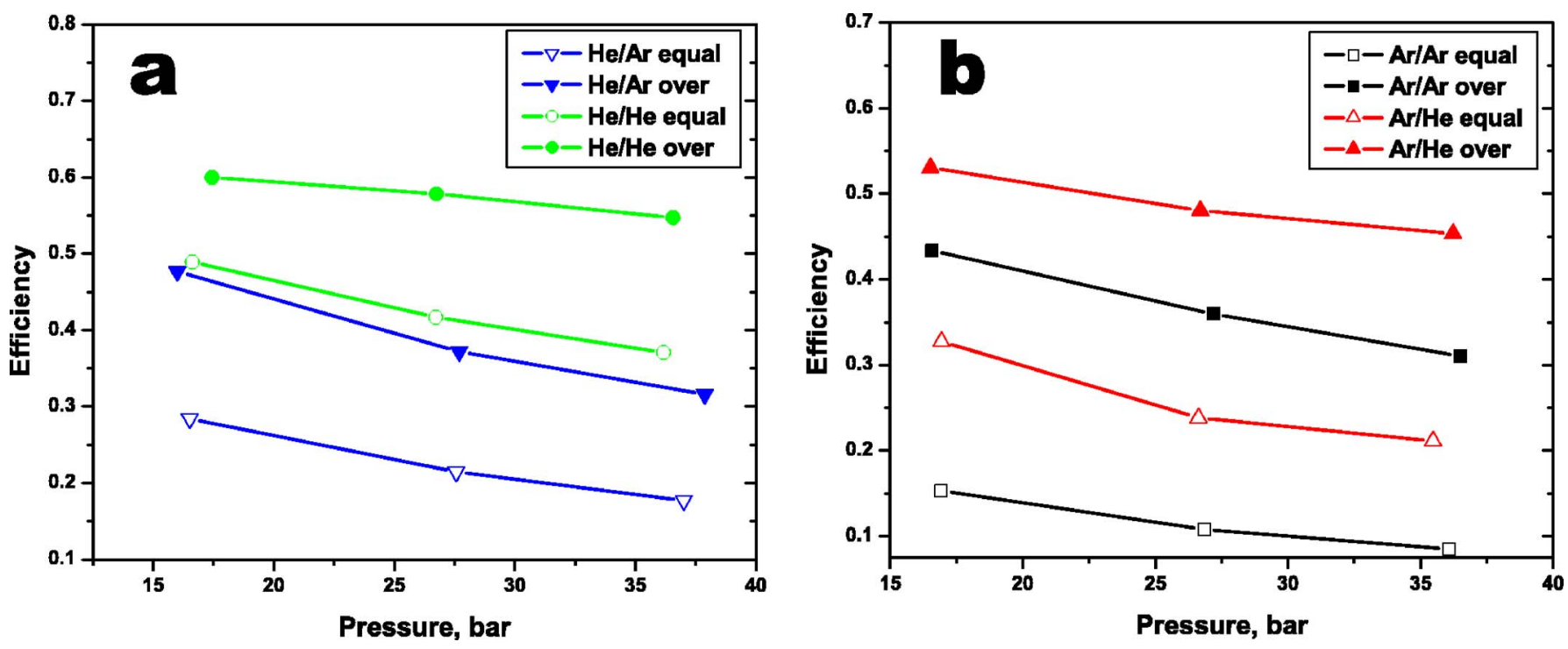

FIG. 7. Efficiency of the valve as a function of the backpressure for different gas combinations. (a) He as working gas and (b) Ar as working gas. The notation coincides with that of Fig. 6.

tion time and efficiency behaviors makes it clear that the prevailing role is played by the change of the injection time. The modification of the decay rate does take place but amounts only to the relatively low value of $20 \%$ to be discussed later. We note also the simple relation between the total number of the injected particles also known as throughput and the efficiency: Th=Ef $p_{w}(0) V$. The maximum realized throughput is about 7.5 bar 1 for argon and about 9.5 bar 1 for helium.

To stay in the framework of the introduced model the decay rate is found from the experiment as

$$
\alpha=\frac{-\ln \left(\frac{p_{w}(\infty)}{p_{w}(0)}\right)}{t^{*}}=-\frac{\ln (1-\mathrm{Ef})}{t^{*}} .
$$

The dependence of the decay rate on the main parameters is illustrated in Fig. 8(a), the error is about 5\%. As expected for the given experimental conditions (the working gas, the backgas, and the overpressure factor) the decay rate is a very weak function of the pressure, which confirms the validity of the used model. The still persisting pressure dependence is caused by the change of the piston stroke as the pressure changes. The abrupt drop at $p=35$ bar for two points arises from the malfunction of the sealing separating back and working chambers, this fact could be already inferred from the corresponding injection duration measurements [Fig. 6(b)]. The experimentally found decay rates differ by a factor of 2-2.5 from the ideal estimation given earlier, while the ratio of $\mathrm{He}$ to Ar decay rates constituting 2.7 coincides with the sound velocity ratio within the accuracy of $20 \%$. This discrepancy is to be attributed to the flow dynamics effects. The further clarification requires a detailed modeling of the outflow process.

It is to be noticed that the decay rate defined in such way is an average over the whole piston motion. The mean decay rate coincides to the first order of accuracy with the value at the average opening

$$
\langle\alpha(h)\rangle=\frac{1}{t^{*}} \int_{0}^{t^{*}} \alpha[h(t)] d t \approx \alpha(\langle h\rangle) .
$$

In assessing the peak valve performance it is necessary to analyze the difference between the maximum and the average values. The in-depth study based on the knowledge of function $\alpha(\langle h\rangle)$ (Fig. 9) and on the measurements of the maximum opening leads to the conclusion that these changes remain at the insignificant several percent level for all cases with the exception of "Ar/Ar equal" and "He/Ar equal." In these two sets the maximum outflow should exceed for a short period of about $1 \mathrm{~ms}$ the average one by $20 \%$. If the specified time is enough for the flow to form, this factor brings the maximum ratings of "*/Ar equal" set close to that of the "*/Ar over." Nevertheless the shown values are with good accuracy representative of the valve functionality. The use of helium in the back section is able to modify the decay rate by about $20 \%$.

The main reason of the low sensitivity of $\alpha$ to the substantial change of $h$ in using helium in the back section is the saturation demonstrated in Fig. 9. When the surface area of the cylinder with the height $h$ based on the orifice of radius $r$ becomes comparable with the value $\pi r^{2}$, or $h=r / 2$ $\approx 2.5 \mathrm{~mm}$, the flow is limited by the orifice. This moment is marked with the black vertical line in Fig. 9. In order to use the piston stroke effectively the orifice is to be extended by a factor of about 2, which corresponds to the four times increase of the decay rate. The further modification of the diameter should cause only a linear effect on $\alpha$ since the flow would be limited by the opening width. A scrupulous analysis taking into account the flow dynamics as well as the piston mass modification is left for a future work.

The other important value intimately related to the decay rate is the ideal particle influx. That is the particle influx rate of the gas into the plasma if the valve were installed next to the plasma edge. The maximum influx is given by $V p_{w}(0) \alpha$. As the average opening is achieved on a timescale $0.5 \mathrm{~ms}$ 

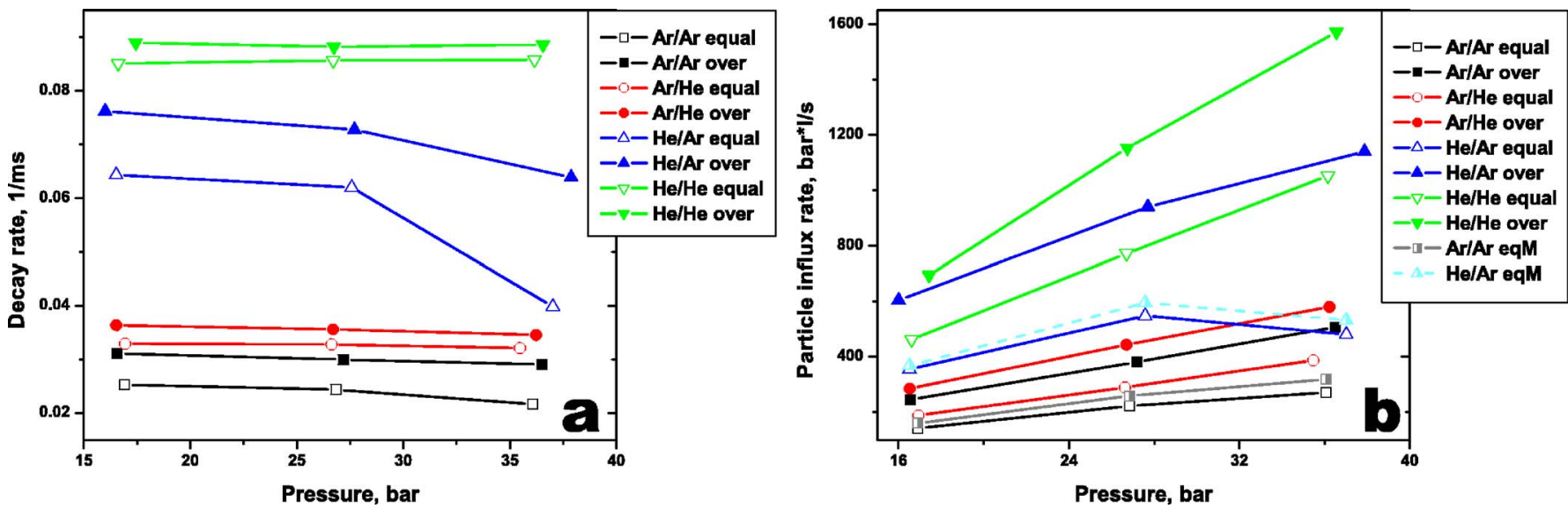

FIG. 8. Time characteristics of the valve. (a) decay rate and (b) ideal particles influx rate. The notation coincides with that of Fig. 6 . The curves marked $M$ in the (b) plot represent the estimation of the maximum outflow rate with the 1.2 modification factor derived.

being much shorter than the exhaust time, the use of $p_{w}(0)$ is justified with the accuracy better than $5 \%$. The values expressed in bar $1 \mathrm{~s}^{-1}$ are shown in Fig. 8(b). Together with the usual data set the outflow rates for the cases Ar/Ar equal and $\mathrm{He} / \mathrm{Ar}$ equal modified by the factor 1.2 discussed ealier are given. The maximum achieved delivery rates are

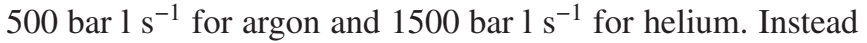
of looking at maximum ratings one can also be interested in the delivery rate averaged over $t^{*}:\left[p_{w}(0)-p_{w}(\infty)\right] / t^{*}$. The transition between this two definitions is straightforward, the correction factor being given by $\mathrm{Ef} /\left(\alpha t^{*}\right)$, which is effectively in the range $0.5-1$ in the present measurements.

\section{CONCLUSIONS}

The piston motion was observed by means of the fast framing camera system and the results were discussed in detail. The piston dynamics was found to strongly depend on the operational pressure and the gases used in both sections. An increase of the backpressure $\left(p_{w}=p_{b}\right)$ decreases the opening width and shortens the injection duration, while the over- pressure in the working chamber and the use of helium in the back section act in the opposite direction, i.e., the injection time and the piston stroke are increased. The advantage of using helium as a closing gas was proposed to be related to the gas dynamics in the back section. Injection durations were measured for all experimental conditions and are in the range 5-20 ms. These data were used to reconstruct the gas outflow rates. It was also found that the valve reaction time is about $0.3 \mathrm{~ms}$ for all cases.

The results of the piston dynamics observations were used to derive the decay rate, the ideal particle influx rate, and to explain tendencies observed in the behavior of the efficiency. The efficiency decreases with the pressure and is increased by the overpressure and the use of He in the back section. The leading role in these modifications is played by the change of the injection duration. The improvement of the decay rate with $\mathrm{He}$ in the back section amounts to about $20 \%$. The low sensitivity of the decay rate to the substantial increase of the opening gap is due to the flow limitation by the output orifice. The decay rate was measured to be
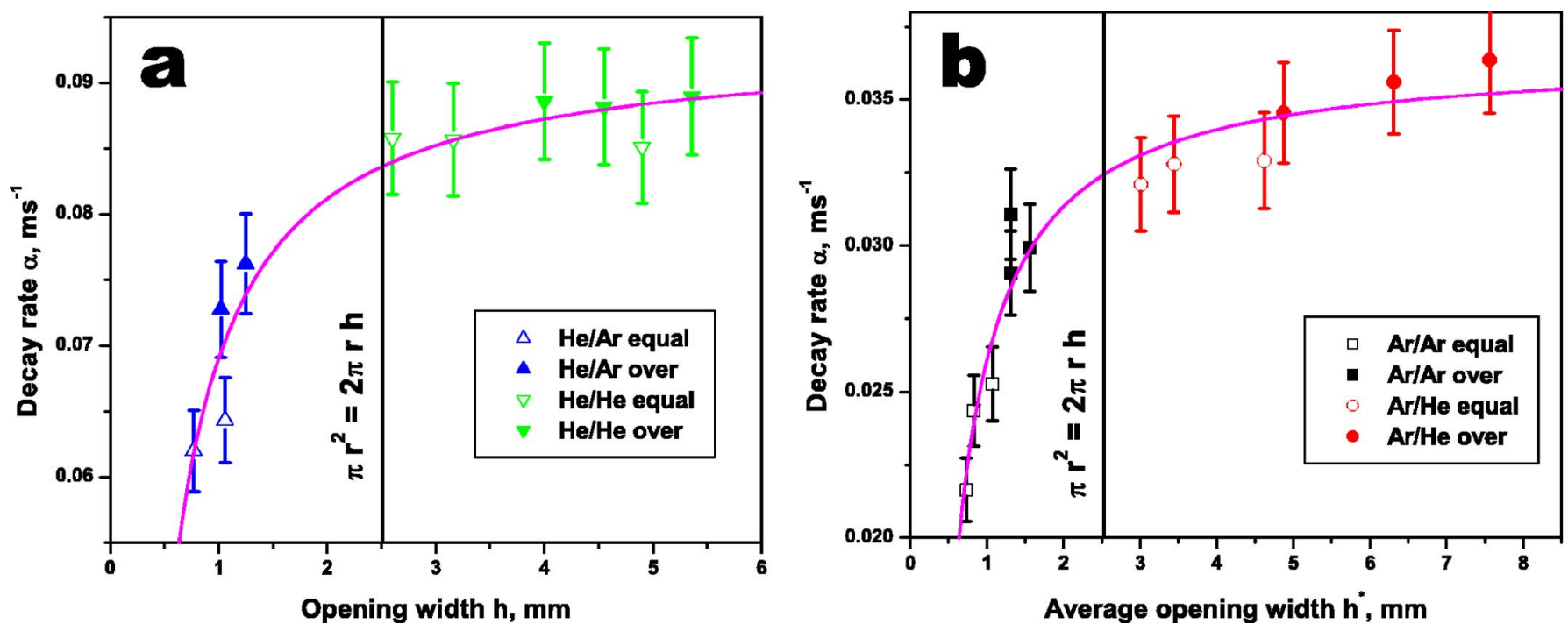

FIG. 9. The dependence of the average decay rate on the average opening width. The fitting curve has the form $a+b / x$. (a) He as working gas and $a$ $\approx 0.093 \mathrm{~ms}^{-1}, b \approx-0.0243 \mathrm{~mm} \mathrm{~ms}^{-1}$ (b) Ar as working gas, $a \approx 0.037 \mathrm{~ms}^{-1}, b \approx-0.0105 \mathrm{~mm} \mathrm{~ms}^{-1}$. The black vertical line marks the opening from which the gas flow is limited by the orifice surface area. 
$0.035 \mathrm{~ms}^{-1}$ for Ar and $0.085 \mathrm{~ms}^{-1}$ for $\mathrm{He}$, or about 12 and 33 $\mathrm{ms}$ in terms of the exhaust times. The expected particle fluxes were also given.

As a summary, the valve on hand can be characterized by the following main parameters: (1) reaction time is about $0.3 \mathrm{~ms}$; (2) the maximum achieved throughput is 7.5 bar 1 for argon and 9.5 bar 1 for helium; (3) the peak delivery rate is 500 bar $1 \mathrm{~s}^{-1}$ for Ar and 1500 bar $1 \mathrm{~s}^{-1}$ for He.

The presented material is supposed to be used as a calibration for DMV experiments at TEXTOR and JET differing only by the value of the volume $\left(V_{\mathrm{JET}}=650 \mathrm{ml}, V_{\mathrm{TEXTOR}}\right.$ $=30 \mathrm{ml}$ ). The reaction time and the particle flux do not depend on the volume and are thought to be the same. This statement is true as long as the characteristic volume length is much larger than the opening gap, which holds for all considered valves. The decay rate $\alpha$, on the other hand, is expected to be reciprocal to the volume, i.e., $\alpha$ is two times smaller for the JET valve and ten times higher for the TEXTOR one. The efficiency and throughput are usually measured in every experiment, e.g., the maximum JET throughput is about 15 bar 1 . The reasons of the efficiency modifications under different experimental conditions are essentially the same as discussed here. Given the efficiency and the scaled decay rate, the injection time can be estimated by using the simple relation $\mathrm{Ef}=1-\exp \left(-\alpha t^{*}\right)$. The influence of the delivery system in each experiment is still to be carefully analyzed. In TEXTOR experiment the delivery tube is so short ( $L=0.8 \mathrm{~m}, d=40 \mathrm{~mm}$ ) as to hardly affect the given values. For JET experiment this analysis seems to be vital as the delivery tube of the same diameter is $4 \mathrm{~m}$ long.

If the high $Z$ impurities like Ar are to be injected with the higher rates the option of mixing with deuterium is to be considered. This option combines the advantages of the high influx rate and the good cooling rate. The additional way to win the particle flux applicable for the next generation valves is to optimize the orifice size. Taking into account the observed piston dynamics, the two times increase of the diam- eter can be recommended. Such diameter increase should lead to four times increase of the gas outflow rate. In other words, the ideal particle influx rate is expected to be about 6000 for $\mathrm{He}$ and 2000 for Ar. The further diameter modification should lead only to the linear increase of the particle flux since the flow would be limited by the piston stroke. The detailed investigation is left for future work.

To a first approximation, the valve with an increased orifice would inject in 10-20 ms the amount of gas between 30 and 60 bar 1 . The system consisting of 4 such valves could provide the total amount of the injected gas more than 100 bar 1 thought to be required for ITER. At the same time the system consisting of four valves deposits gas with higher toroidal symmetry, which is preferable for the mitigation efficiency.

${ }^{1}$ V. Riccardo and JET EFDA contributors, Plasma Phys. Controlled Fusion 45, A269 (2003)

${ }^{2}$ ITER Physics Basis, Nucl. Fusion 39, 3 (1999).

${ }^{3}$ S. C. Bates and K. H. Burrell, Rev. Sci. Instrum. 55, 934 (1984).

${ }^{4}$ V. A. Soukhanovskii, H. W. Kugel, R. Kaita, R. Majeski, and A. L. Roquemore, Rev. Sci. Instrum. 75, 4320 (2004).

${ }^{5}$ E. M. Hollmann, et al., Nucl. Fusion 45, 1046 (2005).

${ }^{6}$ D. G. Whyte et al., Phys. Rev. Lett. 89, 055001 (2002).

${ }^{7}$ D. G. Whyte, 33nd EPS Plasma Physics Conference, Rome, 2006; http:// eps2006.frascati.enea.it/presentazioni_finali/Aula_Mayor/Thursday/ Whyte/EPS_06_whyte.pdf

${ }^{8}$ G. Martin, F. Sourd, F. Saint-Laurent, J. Bucalossi, L. G. Erikson, 32nd EPS Plasma Physics Conference, Tarragona, 2005; http://wwwpub.iaea.org/MTCD/Meetings/PDFplus/fusion-20-preprints/EX_106Rc.pdf

${ }^{9}$ S. L. Milora, S. K. Combs, C. R. Foust, Rev. Sci. Instrum. 57, 2356 (1986).

${ }^{10}$ S. K. Combs, C. R. Foust, and M. J. Gouge, Rev. Sci. Instrum. 75, 270 (2004).

${ }^{11}$ A. Savtchkov, K. H. Finken, G. Mank, Rev. Sci. Instrum. 73, 3490 (2002).

${ }^{12}$ K. H. Finken, G. Mank, A. Krämer-Flecken, and R. Jaspers, Nucl. Fusion 41, 1651 (2001)

${ }^{13}$ A. Savtchkov, Ph.D. thesis, Mathmatisch-Naturwissenschaftlichen Fakultät der Heinrich-Heine Universität Düsseldorf, 2002.

${ }^{14}$ www.prinsci.com/ffcam 